\title{
The Singular Manifold Method: Darboux Transformations and Nonclassical Symmetries
}

\author{
P.G. ESTÉVEZ and P.R. GORDOA \\ Area de Física Teórica, Facultad de Ciencias, \\ Universidad de Salamanca, 37008, SALAMANCA, Spain
}

\begin{abstract}
We present in this paper the singular manifold method (SMM) derived from Painlevé analysis, as a helpful tool to obtain much of the characteristic features of nonlinear partial differential equations. As is well known, it provides in an algorithmic way the Lax pair and the Bäcklund transformation for the PDE under scrutiny.

Moreover, the use of singular manifold equations under homographic invariance consideration leads us to point out the connection between the SMM and so-called nonclassical symmetries as well as those obtained from direct methods. It is illustrated here by means of some examples.

We introduce at the same time a new procedure that is able to determine the Darboux transformations. In this way, we obtain as a bonus the one and two soliton solutions at the same step of the iterative process to evaluate solutions.
\end{abstract}

\section{Introduction}

The advances in the last few years in the field of nonlinear partial differential equations have been both exceedingly relevant and extremely impressive [21].

The study of similarity reductions for PDE's that can be solved by IST led Ablowitz, Ramani and Segur [2] to formulate what we know as the ARS conjecture: Every ordinary differential equation that can be obtained as the similarity reduction of a PDE solvable by IST has, up to a change of variables, the PP [33]. This conjecture provides a necessary condition for checking whether a PDE is integrable or not. Mc Leod and Olver [28] have tested some weak versions of this conjecture. Although there is no rigorous proof of it, the many studies dealing with similarity reductions for integrable PDE's seem to point to its validity as a method of integrability.

The generalization of the PP for PDE's was rigorously established by Weiss, Tabor and Carnevale [36] in a widely recognized paper in which they also developed an elegant formalism: The Singular Manifold Method (SMM) that we will consider here in detail $[37,38]$. It allows to obtain the celebrated Bäcklund transformations and Lax pair in a straightforward and systematic manner. There have also been relationships established between the Singular Manifold Method and $\tau$-functions of the Hirota formalism $[12,18$, 35]. 
One different but not unrelated approach to integrability of PDE's begins with the seminal contribution of Lie in classical symmetries of differential equations, which has been generalized thanks to the work of Bluman and Cole [6] and Olver and Rosenau [30, $31]$, and Fushchich et al $[15,16]$. This new procedure deals with symmetries that leave invariant just a subset of all the possible solutions of the PDE under scrutiny $[15,16]$. These symmetries, which do not form a group in the Lie sense, appear however to be extremely interesting for analyzing the integrability and properties of the PDE as well as its relationship with the PP.

There exists another possibility to analyze nonlinear PDE's. It consists on finding symmetries not through the usual group-theoretical procedures but rather through the so-called Similarity Transformations. Usually one chooses an ansatz $[15,16]$ that allows one to find a change of variables connected with the symmetries of the equation such that the PDE is reduced to an ODE which can be analyzed through the usual Painlevé tests for ordinary nonlinear differential equations. The question of how to choose an adequate set of ansätze leading to the right change of variables (the similarity transformations) has extensively been analyzed by Clarkson and Kruskal [8]. A great deal of work and thought has been dedicated in the last years to the question of what conditions a given nonlinear PDE has to meet in order that the ansatz methods and the nonclassical method would yield the same information. A related question $[13,34]$ is also whether it is possible to generalize the ansatz methods in order that all the similarity transformations could be obtained without using group theory. Our purpose here is to combine the direct method (DM) and symmetry methods with Painlevé ideas. In fact, Cariello and Tabor made the first crucial observation [7] that the DM and the SMM were almost identical when one uses the singular manifold as a reduced variable. Furthermore, as it will be shown, the form for solutions of the singular manifold is proven to be crucial to the answer on the question of why the direct and nonclassical methods should (or should not) yield the same information.

The plan of this paper will be the following. Section $\S 2$ is wholly devoted to an exposition, along general lines, of the Singular Manifold Method and its relationship with the nonclassical symmetries. We will show in section $\S 3$ how this procedure provides an extremely helpful tool to derive Bäcklund Transformations, Lax pair, Darboux Transformations and soliton solutions for the Korteweg-de Vries equation. The rest of the paper constitutes the application of the same techniques to different PDE's including PDE's with the conditional Painlevé property.

\section{The Singular Manifold Method}

\section{2a) Truncated expansion and singular manifold equations}

According to the generalization for PDE of the PP carried out by Weiss, Tabor and Carnevale [36], we require that the solutions of such PDE be written in the form

$$
u=\sum_{j=0}^{\infty} u_{j}(x, t)[\phi(x, t)]^{j-\alpha},
$$

where $\phi(x, t)$ is an arbitrary analytic function depending on the initial conditions that 
will be called the Movable Singularity Manifold henceforth. The $u_{j}(x, t)$ represent analytic functions that will be determined in terms of $\phi$ through the recurrence relationship obtained from substitution of (2.1) on the correspondent PDE. This partial differential equation is said to enjoy the Painlevé property if $u$ is single-valued in the movable singularity manifold, which requires $\alpha$ be a positive integer. The algorithmic procedure used to determine whether a given PDE possesses the PP is quite well known (see [37] and [38]). For us it is more important here to concentrate on the so called Singular Manifold Method which emphasizes only those solutions which arise in truncation of the series (2.1),

$$
u=\sum_{j=0}^{\alpha} u_{j}(x, t)[\phi(x, t)]^{j-\alpha}=u_{0} \phi^{-\alpha}+u_{1} \phi^{1-\alpha}+\ldots . .+u_{\alpha},
$$

where $\phi$ is no longer an arbitrary function but the singular manifold [37] which makes (2.2) to hold. At this point, it is extremely useful to introduce the following quantities to be used throughout the paper:

$$
\begin{gathered}
w=\frac{\phi_{t}}{\phi_{x}}, \\
v=\frac{\phi_{x x}}{\phi_{x}}, \\
s=v_{x}-\frac{v^{2}}{2} .
\end{gathered}
$$

From the obvious condition $\phi_{x t}=\phi_{t x}$ we can easily write the following relationships among the quantities $w, v$, and $s$

$$
\begin{gathered}
v_{t}=\left(w_{x}+w v\right)_{x}, \\
s_{t}=w_{x x x}+2 s w_{x}+w s_{x} .
\end{gathered}
$$

To see the importance of these quantities, note that the PP is invariant under homographic transformations of $\phi$ as:

$$
\phi \rightarrow \frac{a \phi+b}{c \phi+d}
$$

It is easy to check that $w$ and $s$ (the schwartzian derivative) are homographic invariants [11] but not $v$. Inserting (2.2) in the corresponding PDE, one can obtain the coefficients $u_{j}$ in terms of $w, s$, and $v$. Also it follows from the substitution that $u_{\alpha}$ is a solution of the PDE which means that the ansatz (2.2) plays the additional role of being an auto-Bäcklund transformation among solutions of the same PDE. Since $u_{\alpha}$ is a solution of the PDE, the singular manifold must verify an additional equation which can always be written as a relationship among the homographic invariants and their derivatives. This last equation and (2.4) define the singular manifold and will be called the singular manifold equations henceforth. The conclusion of this procedure is that we can express the solutions $u$ (we shall drop the $\alpha$-index henceforth) in terms of the singular manifold quantities as:

$$
u=u(v, w, s)
$$

where $w$ and $s$ must satisfy the singular manifold equations. From this it follows that the SMM is not only applicable to totally integrable equations but also to PDE without the full PP. It suffices to analyze what solutions do have such a property through the 
truncation of the series (2.2) even though the complete PDE is not integrable according to the classical Painlevé test.

\section{2b) Homographic invariance and symmetries}

Next we turn our attention to the problem of symmetries in order to establish a link between the two procedures. The infinitesimal form of the Lie transformation of a PDE can be written as:

$$
\begin{aligned}
x^{\prime} & =x+\varepsilon \xi(x, t, u)+O\left(\varepsilon^{2}\right), \\
t^{\prime} & =t+\varepsilon \tau(x, t, u)+O\left(\varepsilon^{2}\right), \\
u^{\prime} & =u+\varepsilon \eta(x, t, u)+O\left(\varepsilon^{2}\right),
\end{aligned}
$$

so that the associated Lie algebra contains vector fields of the form

$$
v=\xi \frac{\partial}{\partial_{x}}+\tau \frac{\partial}{\partial_{t}}+\eta \frac{\partial}{\partial_{u}} .
$$

The classical Lie method to determine the group of symmetries of a given PDE is well known and can be found in various textbooks $[3,6]$. We are more interested in the nonclassical method $([6,15,30])$ which requires that the symmetries should obey the invariant surface condition,

$$
\xi(x, t, u) u_{x}+\tau(x, t, u) u_{t}=\eta(x, t, u)
$$

associated to the vector field $v(2.8)$.

The final step is to find the set $\{\xi(x, t, u ; \tau(x, t, u) ; \eta(x, t, u)\}$ of components of the vector field by using the techniques described in books $[6]$ and papers $([9,10,24,29])$. The method requires one to consider the cases $\tau=0$ and $\tau \neq 0$ separately.

We note however that the nonclassical method deals mainly with symmetries of the solutions not of the PDE $[16,17]$. Our claim is that the nonclassical symmetries are related to the PP because the Lie point symmetries of the solutions of the SMM are precisely nonclassical symmetries. Therefore we need to find the derivatives $u_{x}$ and $u_{t}$ of the solution defined in (2.6) to apply the condition (2.9). At this point the dependence on $v$ of (2.6) appears to be of paramount importance. Since the PP is invariant under homographic transformations, the presence of the singular manifold in (2.9) must be realized just through quantities that are homographic invariants themselves. Consequently $v$ must be eliminated from the equations. If the solution (2.6) depends linearly on $v$ (Burgers or Fitzhugh-Nagumo equations), the solution (2.6) itself can be used to eliminate $v$. On the other hand, if (2.6) depends quadratically on $v$ (for example KdV or Boussinesq equations) then $v^{2}$ could indeed be eliminated but $v$ is still present in the expressions for $u_{x}$ and $u_{t}$.

We are lead to the conclusion that if the dependence on $v$ of (2.6) is nonlinear, the derivatives $u_{x}$ and $u_{t}$ will depend on $v$ which in turn means that the only possibility for (2.9) to depend just on homographic invariants is that $\tau$ must be different from zero. However for linear dependence, one can use (2.6) itself to eliminate the dependence on $v$ of (2.6). Thus, the derivatives $u_{x}$ and $u_{t}$ will not depend on $v$, and (2.9) may contain cases bold with both $\tau$ zero and nonzero, which implies a richer structure of symmetries. In fact, as we shall show in the examples that follow, it will be possible to obtain the differential equations that the vector field components $\{\xi ; \tau ; \eta\}$ obey just from the singular manifold 
equations. This clearly shows the intimate connection between nonclassical symmetries and the SMM. The classification based on the nonlinear or linear dependence of the solutions on the quantity $v$ is the key point to discerning when we shall be able to obtain the same (or less) information from the direct method and the nonclassical method.

In the case in which the singular manifold is characteristic [37] $\left(\phi_{x}=0\right)$, one important point must be emphasized. Note that for $\phi_{x}=0$ the quantities (2.3) are undefined. The appropriate definitions are now:

$$
q=\frac{\phi_{t t}}{\phi_{t}}
$$

and

$$
h=q_{t}-\frac{q^{2}}{2}
$$

where $h$ is now the only homographic invariant. The discussion about the determination of the infinitesimal in terms of just homographic invariants remains valid for the case of the characteristic manifold. So that, the infinitesimal can have dependence on $h$ but not on $q$.

One should add at this point that the singular manifold may exhibit more symmetries than the ones found through the nonclassical method. Lakshmanan and Sahadevan [23] have shown that some ODE's having the PP possess in addition contact symmetries that in turn give rise to first integrals. This result strongly suggests that for the PDE case the PP must be related to the so called Lie-Bäcklund symmetries.

\section{The Korteweg-de Vries equation}

The combined knowledge of the Bäcklund Transformation (2.2) and the singular manifold equations provides a systematic procedure to obtain the Lax pair, Darboux transformation and solutions for the PDE under consideration. To explain it, let us consider the KdV equation in the form:

$$
u_{t}-6 u u_{x}+u_{x x x}=0
$$

The truncated solution for $u$ derived from the SMM could be written [36] as:

$$
u=-2 \frac{\partial^{2}}{\partial x^{2}}(\ln \phi)+u_{2}
$$

Substitution of (3.2) into (3.1) provides the singular manifold equations, which in this case read:

$$
\begin{gathered}
6 u_{2}=w+4 v_{x}+v^{2}, \\
w+s+6 \lambda=0 .
\end{gathered}
$$

\section{3a) Lax pair}

Furthermore, the Riccati equation (3.3b) can be linearized by the following change:

$$
\phi_{x}=\psi^{2} \quad \rightarrow \quad v=2 \frac{\psi_{x}}{\psi}
$$


such that the corresponding linear system is:

$$
\begin{gathered}
\psi_{x x}=\left(u_{2}+\lambda\right) \psi, \\
\psi_{t}=-u_{2 x} \psi+2\left(u_{2}-2 \lambda\right) \psi_{x}
\end{gathered}
$$

whose compatibility condition demands that $u_{2}$ must be a solution of equation (3.1) as long as $\lambda$ is independent of time.

As a result, equation (3.5) represents precisely the Lax pair for $\mathrm{KdV}$, where $\lambda$ plays the role of the spectral parameter of the Inverse Scattering problem [1].

\section{3b) Generation of the solutions. Hirota's Formalism}

However, unlike the IST method, the Lax pair is now used to generate solutions of the equation by an iterative procedure. Indeed, expression (3.2) represents an auto-Bäcklund transformation among solutions of the KdV equation. Knowing the seed solution $u_{2}$, solving (3.5) allows one to obtain $\psi$ and hence the singular manifold from (3.4). The auto-Bäcklund transform (3.2) leads in this way to a new solution for KdV.

In order to generate solutions, if, for example, one starts from the trivial solution $u_{2}=0$ and we set $\lambda=k^{2}$, then the simplest solution for the eigenfunction of the Lax pair will be:

$$
\psi=e^{k\left(x-4 k^{2} t+x_{0}\right)}
$$

Now solving (3.4), we have for $\phi$ :

$$
\phi=1+e^{2 k\left(x-4 k^{2} t+x_{0}\right)}
$$

with the exception of a multiplicative constant, which is irrelevant for the final result.

From here, simple calculation yields a new solution for KdV making use of (3.2):

$$
u(x, t)=-2 k^{2} \operatorname{sech}^{2}\left\{k\left(x-4 k^{2} t+x_{0}\right)\right\}
$$

where $x_{0}$ is an arbitrary integration constant.

The process can be iterated $n$ times, obtaining at each step the corresponding singular manifold $\phi_{i}$. After $n$ iterations, the solution for $n$-solitons can be written as:

$$
\begin{aligned}
u^{n} & =-2 \frac{\partial^{2}}{\partial x^{2}}\left(\ln \phi_{n}\right)+u_{2}^{n-1}= \\
& =-2 \frac{\partial^{2}}{\partial x^{2}}\left(\ln \phi_{n}\right)-\cdots-2 \frac{\partial^{2}}{\partial x^{2}}\left(\ln \phi_{0}\right)+u_{2}=-2 \frac{\partial^{2}}{\partial x^{2}}(\ln \Phi)+u_{2}
\end{aligned}
$$

where $\Phi=\phi_{0} \phi_{1} \cdots \phi_{n}$. The result (3.9) has a direct analogy with the ansatz of the Hirota bilinear method, in this case it being possible to identify $\Phi$ with the $\tau$ function corresponding to $\mathrm{KdV}$ [18].

\section{3c) Darboux transformations}

We shall now see how to establish the connection between the singular manifold method and the Darboux transformations for the $\mathrm{KdV}$ equation [5, 27]. This procedure, as we shall see, will be able to calculate the solution with two solitons simply, by merely considering 
the Lax pair as a system to which the Painlevé analysis can be applied simultaneously with the starting equation. It should be stressed here that the process to be described below, based on [20], has been successfully applied to other systems [14] and is an evident proof of the close relation between the singular manifold, auto-Bäcklund transformations, and Darboux transformations.

For our analysis, we shall consider the KdV equation and its corresponding Lax pair:

$$
\begin{gathered}
u_{t}^{\prime}+u_{x x x}^{\prime}-6 u^{\prime} u_{x}^{\prime}=0, \\
\psi_{x x}^{\prime}=\left(u^{\prime}+\lambda^{\prime}\right) \psi^{\prime}, \\
\psi_{t}^{\prime}=-u_{x}^{\prime} \psi^{\prime}+\left(2 u^{\prime}-4 \lambda^{\prime}\right) \psi_{x}^{\prime},
\end{gathered}
$$

as a set of nonlinear equations coupled in $u^{\prime}$ and $\psi^{\prime}$, for which the corresponding truncated Painlevé expansions, evaluated from an analysis of dominant terms, can be written as:

$$
\begin{gathered}
u^{\prime}=u-2\left(\frac{\phi_{x}}{\phi}\right)_{x}, \\
\psi^{\prime}=\psi+\frac{\Omega}{\phi},
\end{gathered}
$$

where $\phi$ is the singular manifold.

Owing to the actual form of (3.11), the functions $u$ and $\psi$ are solutions of equations (3.10); that is:

$$
\begin{gathered}
u_{t}+u_{x x x}-6 u u_{x}=0, \\
\psi_{x x}=\left(u+\lambda^{\prime}\right) \psi, \\
\psi_{t}=-u_{x} \psi+\left(2 u-4 \lambda^{\prime}\right) \psi_{x} .
\end{gathered}
$$

Additionally, the singular manifold $\phi$ satisfies (in agreement with (3.3)):

$$
\begin{gathered}
u=\frac{1}{2}\left(v_{x}+\frac{v^{2}}{2}-2 \lambda\right), \\
w+s+6 \lambda=0,
\end{gathered}
$$

which can be linearized, as seen above, with the help of the transformation:

$$
\phi_{x}=\varphi^{2}
$$

to give:

$$
\begin{gathered}
\varphi_{x x}=(u+\lambda) \varphi \\
\varphi_{t}=-u_{x} \varphi+(2 u-4 \lambda) \varphi_{x} .
\end{gathered}
$$

From expressions (3.12b-c) and (3.15), it is immediate that $\varphi$ and $\psi$ are eigenfunctions of the same Lax pair for the same potential $u$ but with different spectral parameters $\lambda$ and $\lambda^{\prime}$.

The following step will be to evaluate the equations that should be satisfied by the $p$ function of (3.11b). To do so, by substituting (3.11) in (3.10) one sees that $\Omega$ must be such that it will satisfy:

$$
\Omega\left(\lambda-\lambda^{\prime}\right)=\varphi\left(\varphi \psi_{x}-\psi \varphi_{x}\right)
$$


Finally, inserting (3.16) in (3.11b), one immediately obtains the desired connection with the Darboux transformations:

$$
\phi_{x}^{\prime}=\psi^{\prime 2}=\frac{1}{\phi^{2}}\left|\begin{array}{cc}
\phi^{2} & -\Omega \\
\Omega+2 \phi \psi & \psi^{2}
\end{array}\right|
$$

\section{3d) The Singular Manifold Method, Nonclassical symmetries and Similarity reductions}

As we have shown, the SMM allows one to write a truncated solution as:

$$
u=u_{2}-2\left(\frac{\phi_{x}}{\phi}\right)_{x}
$$

Let us now remember that substitution of (3.18) into (3.1) provides for $u$ (where we have dropped the index 2 for simplicity) the following expression in terms of the singular manifold:

$$
u=\frac{1}{6}\left(w+4 s+3 v^{2}\right)
$$

where $w$ and $s$ are such that:

$$
w+s+6 \lambda=0
$$

Also (using (3.20a)) $w$ must satisfy the following PDE:

$$
w_{t}+w_{x x x}-3 w w_{x}-12 \lambda w_{x}=0 .
$$

As we have already said in $\S 2$, we would like to eliminate $v$ from the equations since it is not an homographic invariant. The result is:

$$
\begin{aligned}
& v^{2}=2 u+w+8 \lambda \\
& v_{x}=u-\frac{w}{2}-2 \lambda
\end{aligned}
$$

We shall also need in the rest of the analysis the quantities $u_{x}$ and $u_{t}$ which with the help of (3.19-22) are easily found to be:

$$
\begin{gathered}
u_{x}=v u-\frac{w v}{2}-\frac{w_{x}}{2}-2 \lambda v \\
u_{t}=2 w_{x} u+w w_{x}-\frac{w_{t}}{2}+v\left[w_{x x}+w u-\frac{w^{2}}{2}-2 \lambda w\right]+8 \lambda w_{x}
\end{gathered}
$$

To establish the relationship between the SMM and the nonclassical symmetries, we first note that the symmetry condition requires that the vector field components $\{\xi(x, t, u) ; \tau(x, t, u) ; \eta(x, t, u)\}$ satisfy the invariant surface condition $([3,6,24,30])$.

$$
\xi(x, t, u) u_{x}+\tau(x, t, u) u_{t}=\eta(x, t, u) .
$$

Comparison of (3.23) and (3.24) shows that $\xi$ and $\tau$ must be such that the result of the substitution of (3.23) into (3.24) should only be dependent upon the homographic 
invariants $w$ and $s$ (actually only $w$ since $s$ can be eliminated with the help of (3.20)). The non appearance of terms depending upon $v$ requires:

$$
\xi\left[u-\frac{w}{2}-2 \lambda\right]+\tau\left[w_{x x}+w u-\frac{w^{2}}{2}-2 \lambda w\right]=0,
$$

or equivalently,

$$
\begin{gathered}
\xi+w \tau=0 \\
w_{x x}=0
\end{gathered}
$$

Note that to obtain nontrivial vector field components, one is forced from (3.26a) to have $\tau \neq 0$. Otherwise $\xi$ would also vanish. Also we have from (3.21)

$$
w_{t}+w_{x x x}-3 w w_{x}-12 \lambda w_{x}=0 .
$$

The simultaneous solution of (3.26a-c) leads to:

$$
w=-\alpha(t) x-\beta(t),
$$

where $\alpha$ and $\beta$ satisfy the following ODE's,

$$
\begin{gathered}
\alpha_{t}+3 \alpha^{2}=0, \\
\beta_{t}+3 \alpha \beta-12 \lambda \alpha=0,
\end{gathered}
$$

whose general solution can easily be found to be:

$$
\begin{gathered}
\alpha=\frac{1}{3\left(t+t_{0}\right)} \\
\beta=\frac{\left(x_{0}+12 \lambda t\right)}{3\left(t+t_{0}\right)} .
\end{gathered}
$$

Finally using (3.27) and (3.29) in (3.24) and (3.26a), we obtain for the vector field components:

$$
\begin{gathered}
\frac{\xi}{\tau}=\frac{\left(x+x_{0}+12 \lambda t\right)}{3\left(t+t_{0}\right)} \\
\frac{\eta}{\tau}=-\frac{1}{3\left(t+t_{0}\right)}(2 \lambda+2 u)
\end{gathered}
$$

These symmetries are exactly the same that those obtained from both the classical Lie and the nonclassical methods ([8] and [22]). Actually, it is well known that the NCM yields no additional reductions for the KdV Equation.

We now turn our attention to the link between the SMM and the direct method of Clarkson and Kruskal [8]. Let us now suppose that what these authors call the reduced variable $z(x, t)$ is such that our singular manifold $\phi$ is just a function on $z(x, t)$. In this way the definitions (2.3) take now the form,

$$
w=\frac{z_{t}}{z_{x}},
$$




$$
v=\frac{z_{x x}}{z_{x}}+z_{x} p(z)
$$

where

$$
p(z)=\frac{\phi_{z z}}{\phi_{z}}
$$

Comparison between (3.27) and (3.31a) leads to a reduced variable of the form

$$
z=\theta(t) x+\sigma(t)
$$

where

$$
\begin{aligned}
& \alpha=-\frac{\theta_{t}}{\theta}, \\
& \beta=-\frac{\sigma_{t}}{\sigma} .
\end{aligned}
$$

Using (3.31) and (3.33) in the equation (3.21) for the singular manifold it is easy to check that $\sigma$ and $\theta$ must verify the equations:

$$
\begin{gathered}
\frac{\theta_{t}}{\theta}=A \theta^{3}, \\
\frac{\sigma_{t}}{\theta}=(A \sigma+B) \theta^{2}+\lambda,
\end{gathered}
$$

while $p(z)$ satisfies

$$
p_{z}-\frac{p^{2}}{2}=-(A z+B) .
$$

Indeed, A and B are arbitrary constants. The reduced variable (3.33a) together with (3.34) coincides exactly with those determined through the direct method in [8]. The solutions for the equation follow easily from the above. Inserting (3.31) in (3.19), we find:

$$
u=\frac{1}{6} \frac{z_{t}}{z_{x}}+z_{x}^{2} \omega(z)
$$

This last equation is the ansatz [15],[16] for the solutions, used in [8]. In our case

$$
\omega(z)=\frac{1}{6}\left(4 p_{z}+p^{2}\right)
$$

The KdV equation has also truncated solutions in terms of a characteristic singular manifold. If $\phi_{x}=0$ [37], the truncated expansion takes the form (see appendix II for the details):

$$
u^{\prime}=u+\frac{u_{0}}{\phi},
$$

where

$$
6 u_{0 x}=-\phi_{t} .
$$

Inserting (3.38) in (3.1), we obtain the following expression for $u$ in terms of the singular manifold:

$$
u=\frac{x}{12} q(t),
$$


where $q(t)$ has been defined in (2.10a). Furthermore the equation for the singular manifold is just:

$$
h=q_{t}-\frac{q^{2}}{2}=0 .
$$

The equations (3.40) constitute a reduction of $\mathrm{KdV}$ that can be interpreted as a particular case of the direct method in which the reduced variable is $t$. (see Lou [25, 26]).

The symmetries of (3.40) can be obtained noting that the derivatives of (3.40) are

$$
\begin{gathered}
u_{x}=\frac{u}{x}, \\
u_{t}=6 \frac{u^{2}}{x},
\end{gathered}
$$

where we have used (3.40a) to eliminate $q$. The expression for the invariant surface condition is:

$$
\xi\left[\frac{u}{x}\right]+\tau\left[6 \frac{u^{2}}{x}\right]=\eta .
$$

There are two different possibilities:

i) $\tau=0$

In such a case the other infinitesimals are (from (3.42)):

$$
\begin{aligned}
\xi & =1, \\
\eta & =\frac{u}{x} .
\end{aligned}
$$

ii) $\tau=1$

Insofar the solution (3.40a) satisfies $u_{x x}=0$, the equation (3.1) can itself be written as

$$
u_{t}-6 u u_{x}=0 .
$$

This expression has exactly the form of (3.42) with

$$
\begin{gathered}
\xi=-6 u, \\
\eta=0 .
\end{gathered}
$$

The symmetry (3.45) is not a solution of the equations of nonclassical symmetries of $\mathrm{KdV}[22]$. Indeed, to obtain these equations, the coefficient in $u_{x x}$ is required be zero. Nevertheless, (3.45) is not a symmetry of (3.1) but of the system:

$$
\begin{gathered}
u_{t}-6 u u_{x}=0, \\
u_{x x}=0 .
\end{gathered}
$$

It follows that we do not longer need to require this coefficient be zero since $u_{x x}$ already vanishes. Therefore (3.45) is nothing but a nonclassical symmetry of the solution (3.40a). 


\section{The Modified Korteweg-de Vries equation}

\section{4a) The Singular Manifold Method}

The Painlevé test for the $\mathrm{mKdV}$ equation

$$
u_{t}+u_{x x x}-6 u^{2} u_{x}=0
$$

has been carried out in [36] and [38]. Truncated solutions are obtained through the autoBäcklund transformation

$$
u^{\prime}=u+\frac{\phi_{x}}{\phi},
$$

where $\phi$ is the singular manifold. The condition for $u^{\prime}$ to be a solution of (4.1) can be expressed as a condition over the homographic invariant $w$ and $s$ in the form:

$$
u=-\frac{v}{2}
$$

and

$$
\begin{gathered}
w+s=0, \\
s_{t}=w_{x x x}+w s_{x}+2 s w_{x} .
\end{gathered}
$$

One can easily eliminate $s$ from (4.4a-b) yielding:

$$
w_{t}+w_{x x x}-3 w w_{x}=0,
$$

which incidentally is the KdV equation. Due to the linear dependence of (4.3) on $v$, one can express $v$ through (4.3) in terms of $u$ :

$$
v=-2 u,
$$

and using (4.4a),

$$
v_{x}=2 u^{2}-w .
$$

As opposed to the $\mathrm{KdV}$ equation, in the $\mathrm{mKdV}$ equation we can eliminate $v$ in calculating the derivatives $u_{x}$ and $u_{t}$ such that these derivatives depend just on $u$ and the homographic invariants. Their expressions are

$$
\begin{gathered}
u_{x}=\frac{w}{2}-u^{2} \\
u_{t}=-w u^{2}+w_{x} u-\frac{w_{x x}}{2}+\frac{w^{2}}{2},
\end{gathered}
$$

where $w$ is a solution of (4.5).

4b) The SMM, Nonclassical symmetries and Similarity reductions

The substitution of (4.7) into the surface invariant condition

$$
\xi(x, t, u) u_{x}+\tau(x, t, u) u_{t}=\eta(x, t, u)
$$


leads to:

$$
\xi\left[\frac{w}{2}-u^{2}\right]+\tau\left[-w u^{2}+w_{x} u-\frac{w_{x x}}{2}+\frac{w^{2}}{2}\right]=\eta .
$$

Now the difference with previous cases lies in the fact that (4.9) does not depend on $v$ and we do not have restrictions on $\xi$ and $\tau$ due to the presence of $v$ in (4.7). However the expressions for $\xi$ and $\tau$ can still be found by remembering that $w$ must satisfy (4.5). To check this, we must distinguish between two separate cases.

i) $\tau=0$

Without loss of generality we can take $\xi=1$. Then the surface invariant condition can be written as

$$
\eta=u_{x}=\frac{w}{2}-u^{2} .
$$

Since $w$ satisfies (4.5), we can obtain the equation verified by $\eta$ just by substituting

$$
w=2\left(\eta+u^{2}\right)
$$

into (4.5). The result is the following PDE (See Appendix I)

$$
\begin{gathered}
\eta_{t}+\eta_{x x x}-6 u^{2} \eta_{x}+3 \eta \eta_{x x u}-12 u \eta^{2}+3 \eta^{2} \eta_{x u u}+\eta^{3} \eta_{u u u}+ \\
+3 \eta_{x} \eta_{x u}+3 \eta \eta_{u} \eta_{x u}+3 \eta \eta_{x} \eta_{u u}+3 \eta^{2} \eta_{u} \eta_{u u}=0 .
\end{gathered}
$$

This last equation is exactly the same one obtained using the nonclassical method with $\tau=0$. The advantage of our procedure is that we obtain as a bonus the solution (4.11) of this equation.

It is important to emphasize that all truncated solutions of the form (4.3) possess this symmetry without any additional restriction on the singular manifold $\phi$. The only condition is that the system (4.4) must hold. The symmetries described in this subparagraph cannot be obtained through the direct method as we shall discuss below.

ii) $\tau \neq 0$

In this case there is no loss of generality by setting $\tau=1$. Thus equation (4.9) reads:

$$
\xi\left[\frac{w}{2}-u^{2}\right]+\left[-w u^{2}+w_{x} u-\frac{w_{x x}}{2}+\frac{w^{2}}{2}\right]=\eta .
$$

This equation is equivalent to an equation for $w_{x x}$ as:

$$
w_{x x}=-2 \eta+\xi\left(w-2 u^{2}\right)-2 w u^{2}+2 w_{x} u+w^{2},
$$

and since $w$ must also verify (4.5), using together (4.5) and (4.14), we can obtain a system of equations for $\xi$ and $\eta$ that reads (see Appendix I):

$$
\begin{gathered}
\eta_{t}+\eta_{x x x}+3 \eta \xi_{x}-6 u^{2} \eta_{x}=0, \\
\xi_{t}+\xi_{x x x}-3 \eta_{x x u}+3 \xi \xi_{x}+12 u^{2} \eta_{x}-3 \eta \xi_{u}-6 u^{2} \xi_{x}+12 u \eta=0, \\
\xi \xi_{u}+18 u^{2} \xi_{u}-3 \eta_{x u u}+3 \xi_{x x u}=0, \\
\xi_{u}=0,
\end{gathered}
$$




$$
\begin{gathered}
\eta_{x u}-\xi_{x x}=0, \\
\eta_{u u}-3 \xi_{x u}=0 .
\end{gathered}
$$

These equations are indeed the ones obtained using the nonclassical method [24] but the fact that they can be obtained combining the SMM with the NCM seems quite remarkable. The solution of (4.15) is simple and indeed contained in [22]. If $\xi$ and $\eta$ are

$$
\begin{gathered}
\xi=-w \\
\eta=w_{x} u
\end{gathered}
$$

then from (4.14) we see that:

$$
w_{x x}=0 .
$$

The integration of (4.16c) using (4.9) gives:

$$
w=-\alpha(t) x-\beta(t),
$$

where $\alpha$ and $\beta$ must satisfy the ODE's:

$$
\begin{aligned}
& \alpha_{t}+3 \alpha^{2}=0, \\
& \beta_{t}+3 \alpha \beta=0,
\end{aligned}
$$

with solution given by:

$$
\begin{aligned}
& \alpha=\frac{1}{3\left(t+t_{0}\right)}, \\
& \beta=\frac{x_{0}}{3\left(t+t_{0}\right)} .
\end{aligned}
$$

Note that (4.16) corresponds to the well known symmetry of MKdV ([21] and [22]); for this equation, the nonclassical symmetries with $\tau \neq 0$ are equivalent to those determined through the classical Lie method.

As has already been mentioned, the symmetries one can obtain using the Direct Method are just those with $\tau \neq 0$. The relationship with the SMM is established as in the previous cases by imposing that the SMM $\phi$ must be just a function of the reduced variable $z(x, t)$ in the form:

$$
z=\theta(t) x+\sigma(t)
$$

such that:

$$
\begin{gathered}
w=\frac{z_{t}}{z_{x}}, \\
v=z_{x} p(z), \\
p(z)=\frac{\phi_{z z}}{\phi_{z}},
\end{gathered}
$$

The obvious identification of (4.17a) with (4.19b) gives:

$$
\alpha(t)=-\frac{\theta_{t}}{\theta},
$$




$$
\beta(t)=-\frac{\sigma_{t}}{\theta} .
$$

Using (4.19) in the singular manifold equations (4.4), one can conclude that $\theta$ and $\sigma$ must satisfy

$$
\begin{gathered}
\frac{\theta_{t}}{\theta}=A \theta^{3}, \\
\frac{\sigma_{t}}{\theta}=(A \sigma+B) \theta^{2},
\end{gathered}
$$

with A and B arbitrary constants such that:

$$
p_{z}-\frac{p^{2}}{2}=-(A z+B) .
$$

The reduction ansatz can be found upon substitution of (4.19) into the general form of the truncated solution (4.3). We obtain

$$
u=z_{x} \omega(z),
$$

where in our case

$$
\omega(z)=-\frac{p}{2} .
$$

Both the reduction ansatz (4.22) and the reduced variable (4.19a) together with the equations (4.21) are the results of the Direct Method obtained in [8].

We close this example by pointing out that for MKdV, there are not truncated solutions with $\phi_{x}=0$.

\section{Diffusion equation in two dimensions}

The diffusion equation

$$
u_{t}=\frac{1}{r}\left[r\left(\frac{u_{r}}{u}\right)\right]_{r}
$$

is a particular case of the general radially symmetric diffusion equations considered in [19]. We shall use this example to illustrate the case of an equation which does not possess the PP, but still the SMM can be used to analyze both truncated solutions and nonclassical symmetries.

\section{5a) The Singular Manifold Method}

As we have already pointed out, (5.1) does not possess the PP. However one can find solutions with the PP. In order to do this, one should use the following truncation ansatz:

$$
u=-\frac{\phi_{r}^{2}}{\phi_{t} \phi}
$$

which gives rise to the singular manifold equations of the form:

$$
\begin{gathered}
v_{r}-\left(\frac{w_{r}}{w}\right)_{r}-\frac{1}{r} \frac{w_{r}}{w}+\frac{v}{r}=0, \\
\frac{w_{t}}{w^{2}}-\frac{w_{r}}{w}+\frac{1}{r}=0,
\end{gathered}
$$




$$
v_{t}=\left(w_{r}+w v\right)_{r} .
$$

These equations form an overdetermined system whose solution can be found easily to be:

$$
\begin{gathered}
w=r \beta(t)(a \ln r+b), \\
v=\frac{w_{r}}{w}+\frac{\alpha(t)}{r},
\end{gathered}
$$

where $a$ and $b$ are arbitrary constants, and where $\alpha(t)$ and $\beta(t)$ must satisfy the following first-order system of ODE's:

$$
\begin{gathered}
\beta_{t}=a \beta^{2}, \\
\alpha_{t}=a \beta(\alpha+2) .
\end{gathered}
$$

In order to obtain $u_{r}$ and $u_{t}$, we differentiate (5.2) and next we use (5.3) and (5.4) to eliminate $v$. The result is:

$$
\begin{gathered}
u_{r}=\left(\frac{\alpha}{r}\right) u+w u^{2}, \\
u_{t}=\left(w_{r}+(\alpha+1) \frac{w}{r}\right) u+w^{2} u^{2} .
\end{gathered}
$$

\section{5b) The SMM, Nonclassical symmetries and Similarity reductions}

Inserting, as usual, equations (5.6) into the invariant surface condition, one easily finds:

$$
\xi(r, t, u)\left[\left(\frac{\alpha}{r}\right) u+w u^{2}\right]+\tau(r, t, u)\left\{\left[w_{r}+(\alpha+1) \frac{w}{r}\right] u+w^{2} u^{2}\right\}=\eta(r, t, u),
$$

and since the above expression is independent of $v$, one should distinguish as usual among the $\tau=0$ and the $\tau \neq 0$ cases.

i) $\tau=0$

We set $\xi=1$ and then (5.7) yields

$$
\eta=u_{r}=\left(\frac{\alpha}{r}\right) u+w u^{2} .
$$

Let us obtain separately the equations for the nonclassical symmetries using just group theory ( refs. [6],[30]). The result is:

$$
\begin{gathered}
u^{3} \eta_{t}=u^{2} \eta_{r r}+2 u^{2} \eta \eta_{r u}+\frac{1}{r} u^{2} \eta_{r}-\frac{1}{r^{2}} u^{2} \eta+u^{2} \eta^{2} \eta_{u u}- \\
-3 u \eta \eta_{r}-2 u \eta^{2} \eta_{u}-\frac{1}{r} u \eta^{2}+2 \eta^{3}
\end{gathered}
$$

It is an easy task to check that (5.8) is precisely the solution of (5.9) when $w$ is given by (5.4a). Therefore the solutions of the form (5.2) possess a nonclassical symmetry given by (5.8).

ii) $\tau \neq 0$

Setting now $\tau=1$, we obtain $w_{r}$ from (5.7) in the form:

$$
w_{r}=\frac{\eta}{u}-\left(w u+\frac{\alpha}{r}\right) \xi-w^{2} u-\frac{w}{r}(\alpha+1) .
$$


In addition, $w$ must satisfy the singular manifold equations (5.3b). Inserting (5.10a) into this last equation, one obtains:

$$
w_{t}=\frac{w}{u} \eta-w\left(w u+\frac{\alpha}{r}\right) \xi-w^{3} u-\frac{w^{2}}{r}(\alpha+2) .
$$

The compatibility condition $w_{t r}=w_{r t}$ between (5.10a) and (5.10b) forces $\xi$ and $\eta$ be of the form

$$
\begin{gathered}
\xi=-w=-r \beta(t)[a \ln r+b] \\
\eta=\xi\left(\frac{\alpha}{r} u+w u^{2}\right)+\left(w_{r}+(\alpha+1) \frac{w}{r}\right) u+w^{2} u^{2}=\beta(2 a \ln r+2 b+a) u,
\end{gathered}
$$

where

$$
\beta_{t}=a \beta^{2}
$$

If we now look for the equations of the nonclassical symmetries obtained by using solely the group-theoretical non-classical method, we obtain:

$$
\begin{gathered}
u \xi_{u u}+\xi_{u}=0 \\
\eta_{u}-2 u^{2} \xi \xi_{u}+\left[2 \xi_{r u}-\eta_{u u}-\frac{2}{r} \xi_{u}\right] u-\frac{\eta}{u}=0 \\
{\left[-\xi_{t}-2 \xi \xi_{r}+2 \eta \xi_{u}\right] u^{2}+\left[\xi_{r r}-2 \eta_{r u}-\frac{1}{r} \xi_{r}+\frac{1}{r^{2}} \xi-\eta \xi\right] u+2 \eta_{r}=0} \\
{\left[\eta_{t}+2 \eta \xi_{r}\right] u+\left[-\eta_{r r}-\frac{1}{r} \eta_{r}+\eta^{2}\right]=0}
\end{gathered}
$$

One can conclude without too much effort that the solutions of (5.12) are given by:

$$
\begin{gathered}
\xi=-r \gamma(t)[a \ln r+b], \\
\eta=\gamma(t)[2 a \ln r+2 b+2 a-c] u,
\end{gathered}
$$

where

$$
\gamma_{t}=c \gamma^{2}
$$

The solution (5.11) obtained through the SMM corresponds to (5.13) in the particular case in which $c=a$. We shall see below that this case is the only, in which the associated similarity reduction leads to an ODE of the Painlevé type.

Next we shall try to establish a relationship between singular manifold equations and the reduction ansatz of the direct method. Let us assume as always that the singular manifold $\phi$ depends just on the reduced variable $z$. The truncation ansatz leads to

$$
u=\frac{z_{r}^{2}}{z_{t}} p(z)
$$

where

$$
p(z)=-\frac{\phi_{z}}{\phi} .
$$


Aside from the ansatz, $w$ given by (5.4a) leads to a reduced variable that takes the form:

$$
z=\theta(t)[a \ln r+b]
$$

where

$$
\frac{\theta_{t}}{\theta}=a \beta(t),
$$

and $\beta(t)$ can be determined from (5.11). It is not hard to check using the reduction ansatz (5.14) in (5.1) that the resulting ODE satisfied by $p(z)$ must be of the form

$$
\left(\frac{p_{z}}{p}\right)_{z}-p_{z}+\frac{2}{z^{2}}=0
$$

which is an ODE of the Painlevé type.

The reductions associated to the symmetries (5.13) are also of the form (5.14), but in this case $\theta(t)$ is given by $\frac{\theta_{t}}{\theta}=a \gamma(t)$ where $\gamma(t)$ is defined in (5.13c). One can easily check that the reduced equation is now

$$
\left(\frac{p_{z}}{p}\right)_{z}-p_{z}+\left(\frac{c}{a}-1\right) p+\frac{2}{z^{2}}=0
$$

This is not an ODE of the Painlevé type except in the case $c=a$ which is precisely the one determined by the SMM.

\section{Conclusions}

We do not know whether all this analysis will eventually become a set of theorems proving with all possible generality the detailed properties that make the SMM (based upon the Painlevé property) an invaluable tool for analyzing partial differential equations, its symmetries, and reductions ansatzë for its solutions. An interesting point of view which presents a new perspective in the link between symmetries and similarity reductions has recently put forward by Olver [32] and Arrigo and Broadbridge [4]. Awaiting for a more comprehensive formal theory, we still hope in the field of Applied Mathematics to continue our line of research by offering more and more cases of physical interest in which this formalism proves to be both useful and beautiful.

\section{Acknowledgments}

We would like to thank Prof. J. M. Cerveró for encouragement, illuminating discussions, and careful reading of the manuscript. Also we thank J.Villarroel, P. Clarkson and E. Mansfield for interesting suggestions.

This research has been supported in part by DGICYT under project PB92-0302. 


\section{- APPENDIX I: Symmetries of mKdV}

As we have seen in $\S 4$, the cases $\tau=0$ or $\tau \neq 0$ must be discussed separately.

1) $\tau=0$

Substitution of (4.11) in (4.5) yields:

$$
D_{t} \eta+D_{x x x} \eta-6 u^{2} D_{x} \eta-12 u \eta^{2}=0,
$$

where $D$ means a total derivative since

$$
u_{x}=\eta(x, t, u) .
$$

If we now take partial derivatives in (A.I.2) and with the aid of (4.1):

$$
u_{t}=6 u^{2} \eta-D_{x x} \eta,
$$

we finally obtain (4.12).

2) $\tau \neq 0$

From the equations (4.7.a) and (4.7b) it is easy to obtain:

$$
\begin{gathered}
w=2\left(u_{x}+u^{2}\right), \\
w_{x x}=-2 A+4 u u_{x x}+4 u_{x}^{2}+12 u^{2} u_{x},
\end{gathered}
$$

where

$$
A=\eta-\xi u_{x}=u_{t}
$$

Using (A.I.4) and (A.I.5) in (4.5), the result is:

$$
w_{t}=2 D_{x} A+4 u A,
$$

and $u_{t}$ has been eliminated using (A.I.6) and the same has been done for $u_{x x x}$ with the aid of (4.1).

Imposing the compatibility condition $w_{x x t}=w_{t x x}$ between (A.I.5) and (A.I.7) and eliminating $u_{t}$ and $u_{x x x}$ as before, we obtain:

$$
D_{t} A+D_{x x x} A-6 u^{2} D_{x} A-12 u u_{x} A=0,
$$

with $A$ given by (A.I.6). This is the usual equation for the nonclassical symmetries [15]. Now it is necessary to proceed exactly as in the nonclassical method [24] expressing the total derivatives in terms of the partial ones. The result is the equations (4.15).

\section{- APPENDIX II: Characteristic Singular Manifold for $\mathrm{KdV}$}

If the singular manifold is characteristic $\left(\phi_{x}=0\right)$, the truncation of the series (3.1) is:

$$
u^{\prime}=u+\sum_{j=0}^{\alpha-1} u_{j}(x, t)[\phi(t)]^{j-\alpha} .
$$


The substitution of such an expansion in (3.1) provides:

$$
\begin{gathered}
\alpha=1, \\
6 u_{0 x}=-\phi_{t}, \\
u_{0 t}+\left(u_{0 x x}-6 u u_{0}\right)_{x}=0, \\
u_{t}+u_{x x x}-6 u u_{x}=0 .
\end{gathered}
$$

And the integration of (A.II.2b-c) yields :

$$
\begin{gathered}
u_{0}=\frac{-1}{6}\left(\phi_{t} x+m(t)\right), \\
u=\frac{1}{6} \frac{\frac{\phi_{t t}}{2} x^{2}+m_{t} x+n(t)}{\phi_{t} x+m(t)},
\end{gathered}
$$

where $m$ and $n$ are functions of t. Inserting (A.II.3b) in (A.II.2d), we obtain:

$$
\begin{gathered}
h=q_{t}-\frac{q^{2}}{2}=0, \\
\frac{m_{t}}{m}=\frac{q}{2}, \\
n=0 .
\end{gathered}
$$

and $q$ has been defined in (3.10a) . The solution (A.II.3b) leads finally to:

$$
u=\frac{x}{12} q(t),
$$

where $q$ satisfies (A.II.4a). 


\section{References}

[1] Ablowitz M.J. and Clarkson P.A., Solitons, Nonlinear Evolution Equations and Inverse Scattering, Cambridge University Press, 1991.

[2] Ablowitz M.J., Ramani A. and Segur H., Lett. Nuov. Cim., 1978, V.23, 333.

[3] Ames W.F., Nonlinear Partial Differential Equations in Engeneering, Academic Press, New York, 1967.

[4] Arrigo D.J., Broadbridge P. and Hill J.M., J. Math. Phys., 1993, V.34, 4692.

[5] Athorne C. and Nimmo J.J.C., Inverse Problems, 1991, V.7, 645-654.

[6] Bluman G.W. and Cole J.D., Similarity methods for Differential Equations, Appl. Math. Sci., Springer Verlag. Berlin, 1974, V.13.

[7] Cariello F. and Tabor M., Physica D, 1991, V.53, 59.

[8] Clarkson P.A. and Kruskal M.D., J. Math. Phys., 1989, V.30, 2201.

[9] Clarkson P.A. and Mansfield E., Physica D, 1994, V.70, 250.

[10] Clarkson P.A. and Mansfield E., Algorithms for the nonclassical method of symmetry reductions, SIAM J, Appl. Math., 1994 (to appear).

[11] Conte R., Phys. Lett., 1988, V.134, 100.

[12] Estévez P.G., Gordoa P.R., Martínez Alonso L.and Medina Reus E., J. Phys. A, 1993, V.26, 1915.

[13] Estévez P.G., Phys. Lett. A, 1992, V.171, 259,.

[14] Estévez P.G. and Leble S., Acta Applicandae Mathematicae, 1995, V.39, 277-294.

[15] Fushchich W., Nikitin A., Symmetries of Maxwell's Equations, Reidel Pub. Comp., 1987, Appendix 4, 220p. (Russian version, 1983, Naukova Dumka, Kyïv)

Fushchich W.I.,Tsyfra I.M.,On reduction and solutions of the nonlinear wave equations with broken symmetry, J. Phys. A: Math. Gen., 1987, V.20, N 2, L45-L47.

Fushchich W.I. and Zhdanov R.Z., Symmetry and exact solutions of nonlinear spinor equations, Phys. Rep., 1989, V.172, N 4, 123-174.

[16] Fushchich W., Shtelen' W., Serov N., Symmetry Analysis and Exact Solutions of Equations of Nonlinear Mathematical Physics, Dordrecht, Kluwer Academic Publishers, 1993, 430p. (Rusian version, 1989, Naukova Dumka, Kyïv)

Fushchich W., Serov N., The symmetry and exact solutions of the nonlinear multidimensional Liouville, d'Alambert and eikonal equations, Phys. A: Math. Gen., 1983, V.16, N 15, 3645-3658.

Fushchich W., Zhdanov R., On reduction and some new exact solutions of the nonlinear Dirac and Dirac-Klein-Gordon equations, Phys. A: Math. Gen., 1988, V.21, N 1, L5-L9; J. Phys. A 21, L5-L9, (1988).

[17] Gardner C. S., Greene J.M., Kruskal M.D. and R.M. Miura R.M., Phys. Rev. Lett., 1967, V.19, 1095.

[18] Hirota R., in "Solitons", Edited by R.K. Bullough and P.J. Caudrey. Topics in Current Physics, V.17, Springer-Verlag, Berlin, 1980, 157.

[19] King J.R., J. Phys. A, 1990, V.23, 3681.

[20] Konopelchenko B.G. and Stramp W., J. Math . Phys., 1991, V.32, 40-49.

[21] Kruskal M.D. and Clarkson P.A., Stud. Appl. Math., 1992, V.86, 87. 
[22] Lakshmanan M. and Kaliappan P., J. Math. Phys., 1983, V.24, 795.

[23] Lakshmanan M. and Sahadevan R., Physics Reports, 1993, V.224, 1.

[24] Levi D. and Winternitz P., J. Phys. A, 1989, V.22, 291.

[25] Lou S.Y., Phys. Lett. A, 1990, V.151, 133.

[26] Lou S.Y. et al, J. Phys. A: Math. and Gen., 1991, V.24, 1455.

[27] Matveev V.B. and Salle M.A., Darboux Transformations and Solitons, Springer-Verlag, 1991.

[28] McLeod J.B. and Olver P.J., SIAM J. Math. Anal., 1983, V.14, 488.

[29] Nucci M.C. and Clarkson P.A., Phys. Lett. A, 1992, V.164, 49.

[30] Olver P.J. and Rosenau P., Phys. Lett. A, 1986, V.114, 107.

[31] Olver P.J. and Rosenau P., SIAM J. Appl. Math., 1987, V.47, 263.

[32] Olver P.J., Proc. R. Soc. Lond. A 444, 509, (1994).

[33] Painlevé P., Acta Mathematica, París, 1900.

[34] Pucci E., J. Phys. A, 1992, V.25, 2631.

[35] Tabor M. and Gibbon J.D., Physica D, 1986, V.18, 180.

[36] Weiss J., Tabor M. and Carnevale G., J. Math. Phys., 1983, V.24, 522.

[37] Weiss J., Solitons in Physics, Mathematics and Nonlinear Optics, IMA series, 17, Edited by P. Olver and D. Sattinger, Springer Verlag, 1990.

[38] Weiss J., J. Math. Phys., 1983, V.24, 1405. 